\title{
Effet du contexte lexical sur l'accès à la signification d'homographes
}

In: L'année psychologique. 2002 vol. 102, n¹. pp. 31-63.

\section{Citer ce document / Cite this document :}

Thérouanne $\mathrm{P}$., Denhière Guy. Effet du contexte lexical sur l'accès à la signification d'homographes. In: L'année psychologique. 2002 vol. 102, n¹. pp. 31-63.

doi : 10.3406/psy.2002.29581

http://www.persee.fr/web/revues/home/prescript/article/psy_0003-5033_2002_num_102_1_29581 


\section{Résumé}

Résumé

Nous présentons trois expériences de décision lexicale réalisées afin d'étudier l'effet du contexte lexical sur l'accès à la signification des homographes homophones. Dans les deux premières expériences, chaque essai était constitué par la présentation d'un mot contexte, d'un item ambigu, et d'un mot cible. Les résultats ont mis en évidence un effet précoce du contexte sur l'accès à la signification. Alors que la présentation d'un mot contexte relié à la même acception de l'item ambigu que le mot cible entraînait une facilitation relative des temps de décision, un mot contexte relié à une acception différente ne provoquait pas d'inhibition relative du traitement du mot cible. Par ailleurs, cet effet du contexte se manifestait quelle que soit la fréquence relative de l'acception à laquelle était relié le mot cible. La troisième expérience nous a permis d'écarter la possibilité d'un effet direct du mot contexte sur le mot cible. La discussion de ces résultats porte sur la représentation des homographes en mémoire.

Mots-clés : représentation, ambiguïté lexicale, homographes, contexte.

\section{Abstract}

Summary : Lexical context effect on meaning access of homographs

We present three lexical decision experiments investigating the effect of lexical context on homophonic homographs meaning access. In the two first experiments, each trial consisted of the presentation of a context word, an ambiguous word as a prime, and a target word. The context word was related either to the dominant or subordinate meaning, or was not related. The target word was related to the dominant meaning of the ambiguous word in Experiment 1, and to the subordinate meaning in Experiment 2. The presentation time of words and the ISI were also manipulated. The results indicated an early effect of the context on meaning access : whereas the presentation of a context word related to the same meaning as the target word resulted in a facilitation of reaction times, a context word related to a different meaning did not produce an inhibition effect on target processing time. Moreover, this pattern ofresults was obtained whatever the relative frequency of the meaning related to the target word. A third experiment was conducted in order to test the possibility of a direct priming effect between the context and the target words related to the same meaning. Our results are discussed with regard to the hypothesis of competition between the different meanings of homographs.

Key words : representation, lexical ambiguity, homographs, context. 


\title{
EFFET DU CONTEXTE LEXICAL SUR L'ACCÈS À LA SIGNIFICATION D'HOMOGRAPHES
}

\author{
par Pierre ThérociñNe et Guy Denhière
}

\begin{abstract}
Remerciements : Nous tenons à remercier Jonathan Grainger, Bruno Lecoutre, Pierre Marquer et Stéphanie Montoya pour leurs suggestions lors de la réalisation de cette étude, ainsi que Juan Segui et les deux experts pour les remarques constructives formulées sur deux versions antérieures de ce manuscrit.
\end{abstract}

SUMMARY : Lexical context effect on meaning access of homographs

We present three lexical decision experiments investigating the effect of lexical context on homophonic homographs meaning access. In the two first experiments, each trial consisted of the presentation of a context word, an ambiguous word as a prime, and a target word. The context word was related either to the dominant or subordinate meaning, or was not related. The target word was related to the dominant meaning of the ambiguous word in Experiment 1, and to the subordinate meaning in Experiment 2. The presentation time of words and the ISI were also manipulated. The results indicated an early effect of the context on meaning access : whereas the presentation of a context word related to the same meaning as the target word resulted in a facilitation of reaction times, a context word related to a different meaning did not produce an inhibition effect on target processing time. Moreover, this pattern of results was obtained whatever the relative frequency of the meaning related to the target word. A third experiment was conducted in order to test the possibility of a direct priming effect between the context and the target words related to the same meaning. Our results are discussed with regard to the hypothesis of competition between the different meanings of homographs.

Key words : representation, lexical ambiguity, homographs, context.

1. 29. avenue Robert-Schuman, 13621 Aix-en-Provence.

2. E-mail : therouan(wup.univ-mrs.fr, denhiere(a)up.univ-mrs.fr 


\section{INTRODUCTION}

La phase d'accès au lexique mental, au cours de laquelle les représentations correspondant aux mots sont activées, est traditionnellement située entre l'analyse purement perceptive du langage et les aspects plus élaborés de la compréhension. Deux conceptions théoriques s'opposent quant au rôle attribué au contexte, notamment discursif, lors de l'accès au lexique : les conceptions dites « Modulariste »(Forster, 1979 ; Segui, 1992) et «Interactive » (Marslen-Wilson et Tyler, 1987 ; Sharkey, 1990). Selon la première conception, le traitement lexical constitue un module autonome. Les traitements mis en cuvre lors de l'accès au lexique mental ne sont donc pas contraints par le contexte, dont l'intervention est considérée comme postérieure à cette phase d'accès. À l'inverse, la seconde conception postule un degré élevé d'interaction entre les différents processus mis en œuvre lors du traitement du langage et prédit donc un effet précoce du contexte discursif sur l'accès à la signification des mots. L'utilisation de mots homographes (par exemple, « vol ») a constitué un moyen privilégié d'éclaircir le rôle exercé par le contexte lors de cet accès (voir Mullet et Denhière, 1997 ; Rayner, Pacht et Duffy, 1994, pour une revue de ces recherches). Les homographes homophones (ou items ambigus) ont pour particularité de posséder des significations différentes pour une même forme orthographique et phonologique. Cette propriété permet l'étude de l'effet du contexte sur l'accès à des significations indépendantes, tout en neutralisant l'effet des caractéristiques lexicales des mots dont l'accès à la signification est étudié.

Plusieurs recherches (Onifer et Swinney, 1981 ; Seidenberg, Tanenhaus, Leiman et Bienkowski, 1982; Till, Mross et Kintsch, 1988) ont contribué à étayer l'hypothèse du modularisme lexical, en montrant que les différentes acceptions d'un item ambigu sont activées quelle que soit la signification à laquelle est relié le contexte dans lequel il apparaît. Un nombre important d'expériences sont parvenues par la suite à montrer un effet précoce du contexte (Simpson, 1981 ; Tabossi, Colombo et Job, 1987 ; Vu, Kellas et Paul, 1998). Dans les expériences de Vu et al. (1998), des phrases dans lesquelles l'objet du verbe est un ou item ambigu sont présentées mot après mot à l'écran et les 
sujets doivent lire à voix haute un mot cible présenté immédiatement après le mot ambigu. Le temps moyen d'énonciation du mot cible est plus court lorsqu'il est relié à la même acception que celle induite par le contexte que lorsqu'il est non relié. En revanche, aucune différence n'est constatée lorsque le mot cible est relié à une acception différente de celle induite par le contexte. Cet effet du contexte disparaît lorsque les mots de la phrase sont mélangés de sorte que la représentation générale du texte n'induise plus une acception. Les effets d'amorçage obtenus proviennent donc des contraintes exercées par la signification de la phrase, et non des relations entretenues entre les mots formant la phrase et le mot cible.

Ainsi, les résultats de ces différentes recherches ne permettent pas de départager les conceptions modulariste et interactive. Deux remarques importantes doivent être faites sur cette confrontation théorique. La première concerne une troisième conception récemment proposée par Twilley et Dixon (2000). Le modèle d'Activation Parallèle Indépendante élaboré par ces auteurs préserve la modularité des processus, dans la mesure où l'accès au lexique et l'intégration contextuelle s'effectuent de façon indépendante, tout en permettant la simultanéité de ces deux processus. Ainsi, dans ce cadre théorique, des résultats montrant un effet précoce du contexte permettent de rejeter l'hypothèse de la séquentialité des processus, sans pour autant remettre en cause leur indépendance fonctionnelle. Par conséquent, l'étude du décours temporel de l'effet du contexte sur l'accès à la signification des mots ne permet plus de déterminer précisément l'architecture cognitive impliquée dans le traitement du langage. La seconde remarque porte sur la nature même de la représentation des mots homographes en mémoire qui n'est pas à l'heure actuelle clairement définie. Or, la façon dont elle est conçue par les chercheurs peut conditionner l'interprétation des effets de contexte obtenus. Par exemple, Vu et al. (1998) ont montré que la signification contextuellement non appropriée est très rapidement désactivée. En supposant une compétition entre les différentes acceptions d'un item ambigu, ce résultat peut refléter une désactivation de l'acception contextuellement non pertinente suite à l'augmentation de l'activation de l'autre acception par le contexte. Dans ce cas, l'effet du contexte sur l'acception non pertinente s'effectue de façon indirecte. En l'absence d'une telle compétition, c'est alors le traitement de 
l'information contextuelle qui entraîne de façon directe la désactivation de l'acception contextuellement non pertinente (voir Gernsbacher et Faust, 1991 ; Kintsch, 1988 ; Twilley et Dixon, 2000, pour des explications différentes). Par conséquent, en plus de son intérêt propre, déterminer la représentation des homographes apparaît nécessaire pour comprendre pleinement les traitements dont ils font l'objet lors d'une activité de compréhension du discours. L'étude de la représentation des homographes constitue donc l'objet du travail que nous présentons.

\section{LA REPRÉSENTATION DES HOMOGRAPHES}

Une ambiguïté lexicale est constituée par une séquence de lettres ou de phonèmes susceptible de conduire à plusieurs interprétations clairement distinctes. Ce type d'ambiguïté, fréquemment rencontrée dans le discours, constitue une famille relativement disparate et il convient de clarifier brièvement les différences opposant les homographes aux autres types d'ambiguïtés lexicales. Un critère sémantique permet d'opposer les mots polysémiques, qui possèdent plusieurs acceptions que l'on peut mettre en relation, aux homonymes dont les relations sémantiques entre les différentes acceptions ne sont pas aisément identifiables (François, 1994). Différents types d'homonymes peuvent également êtres identifiés selon des critères linguistiques. Contrairement aux homophones hétérographes (par exemple, « champ » et « chant »), les différentes acceptions des homographes ne se distinguent pas par leur forme orthographique. Pour certains homographes, la catégorie grammaticale (par exemple, «boucher ») ou le genre grammatical (par exemple, «moule ») permet de distinguer leurs différentes acceptions (voir Seidenberg et al., 1982; Gardye 2000). Dans d'autres cas, cependant très rares, la forme phonologique permet de différencier les acceptions (par exemple, « reporter »). Enfin, pour un certain nombre de mots homographes (par exemple, " vol »), seul le contexte sémantique dans lequel ils apparaissent permet de décider quelle en est l'interprétation appropriée. Ce sont ces derniers qui ont été le plus souvent utilisés pour confronter les hypothèses modulariste et interactive et ce sont eux dont il sera question désormais. Plusieurs hypothèses psychologiques portant sur la représentation de ces homographes ont été 
envisagées et se distinguent selon deux critères. Le premier concerne le niveau lexical des représentations; la conception selon laquelle chacune des acceptions possède sa propre entrée lexicale est opposée à la conception selon laquelle une entrée lexicale unique est rattachée aux différentes acceptions'. Le second critère concerne le niveau sémantique et correspond à la présence ou l'absence d'une relation inhibitrice entre les représentations correspondant aux différentes acceptions (voir fig. 1).

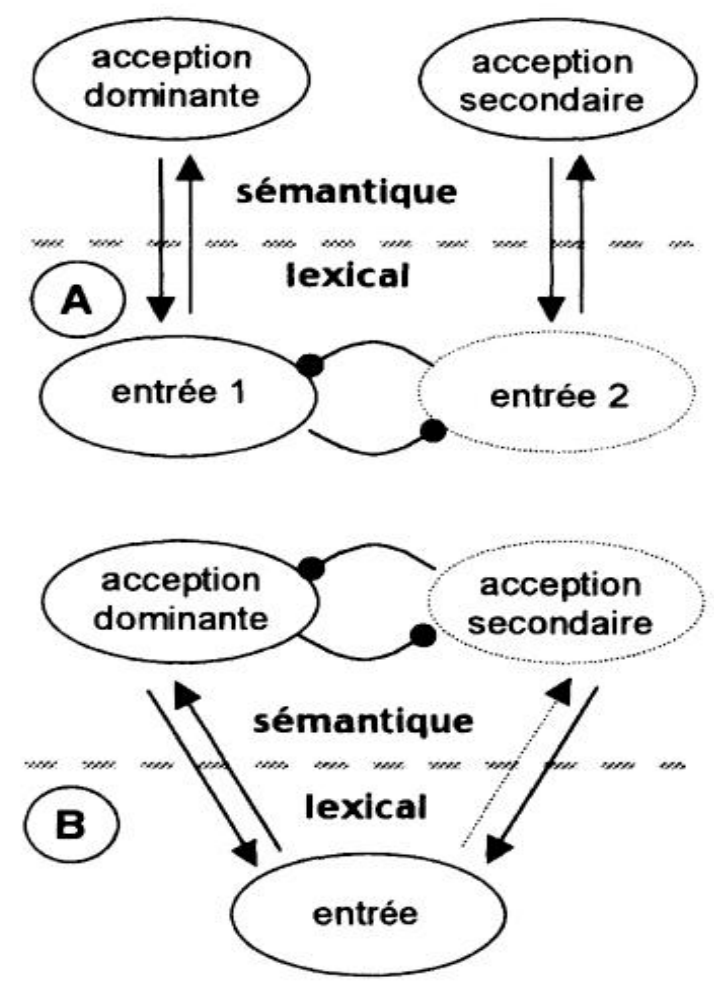

Fig. 1. - Représentation schématique des hypothèses portant sur les représentations lexicales et sémantiques d'un item ambigu. Les flèches indiquent les relations excitatrices et les arcs les relations inhibitrices. $A$ : entrées lexicales multiples. B : entrée lexicale unique. Les relations inhibitrices sont absentes dans l'hypothèse d'une compétition passive entre les entrées lexicales (A) et dans celle de l'absence de compétition entre les deux acceptions (B)

Lexical and semantic representations of an ambiguous word according to the different hypotheses. Arrows represent facilitatory connections and curved lines represent inhibitory connections. A : Multiple lexical entries. B : Single lexical entry. Inhibitory connections are discarded according to the hypothesis of a passive competition between entries $(A)$ and the hypothesis assuming no competition between meanings ( $B$ )

1. L'entrée lexicale correspond à la représentation orthographique et phonologique d'un mot activée par les représentations pré-lexicales des lettres et des phonèmes (Rubenstein et al., 1971). Ainsi, le niveau lexical est à distinguer du niveau sémantique des représentations (Balota et Paul, 1996). 
L'hypothèse des entrées lexicales multiples des homographes (Forster et Bednall, 1976 ; Jastrzembski, 1981 ; Kellas, Ferraro et Simpson, 1988 ; Rubenstein, Lewis et Rubenstein, 1971) a initialement été proposée par Rubenstein et ses collègues pour rendre compte de l'effet d'ambiguïté : les temps de décision lexicale sont plus courts sur les mots homographes que sur les mots non ambigus'. Cet effet est interprété comme reflétant la probabilité plus importante d'identifier une entrée lexicale lorsque plusieurs entrées sont compatibles avec l'analyse perceptive du mot. Cette hypothèse supposant qu'une entrée lexicale différente est associée à chacune des acceptions implique que la distinction entre les acceptions se situe dès le niveau lexical des représentations. Les modèles de la reconnaissance des mots s'accordent sur l'existence d'une compétition entre les entrées lexicales permettant la sélection d'une seule représentation lexicale à l'issue de l'identification du mot. Celleci peut être passive, dans la mesure où l'identification d'une entrée lexicale arrête le processus de reconnaissance du mot et empêche donc l'activation des autres entrées lexicales (Forster, 1976 ; Jastrzembski, 1981). Cette compétition peut également être active dans les modèles postulant que l'ensemble des entrées lexicales sont reliées entre elles par des relations inhibitrices (McClelland et Rumelhart, 1981 ; Grainger et Jacobs, 1996).

Si l'hypothèse des entrées lexicales multiples a été formulée pour expliquer l'effet d'ambiguité, l'hypothèse d'une entrée lexicale commune aux différentes acceptions s'avère également capable d'en rendre compte de la façon suivante : l'entrée lexicale active les deux acceptions qui envoient en retour une quantité d'activation plus importante vers l'entrée lexicale que dans le cas d'un mot ne possédant qu'une seule acception (voir Gottlob, Goldinger, Stone et van Orden, 1999). L'hypothèse d'une entrée lexicale unique se décline en deux versions selon qu'elle est envisagée conjointement à une compétition issue de relations inhibitrices entre les différentes acceptions (Gottlob et al., 1999 ; Kintsch, 1988) ou à l'absence d'une telle compétition (Seidenberg et al., 1982 ; Twilley et Dixon, 2000).

1. L'effet d'ambiguité a été remis en question (Forster et Bednall, 1976 ; Gernsbacher, 1984), puis retrouvé avec un contrôle approprié des stimulus (Kellas et al., 1988 ; Borowsky et Masson, 1996). 
Par conséquent, il n'existe pas de conception consensuelle de la représentation des homographes en mémoire. Sur un plan empirique, l'existence de l'effet d'ambiguïté ne permet pas en soi de départager les différentes hypothèses envisagées. Cependant, d'autres résultats expérimentaux sont à considérer : de nombreuses expériences ont montré que les différentes acceptions d'un item ambigu sont activées après sa présentation en l'absence de contexte (Simpson et Burgess, 1985 ; Marquer, Lebreton, Léveillé et Dioniso, 1990) ou dans un contexte sémantiquement neutre (Vu et al., 1998). Par exemple, Marquer et al. (1990) ont utilisé des mots homographes faiblement et fortement polarisés. $L$ 'adjectif « polarisé » désigne le fait que les acceptions d'un item ambigu sont caractérisées par des fréquences d'utilisation différentes. À chaque item ambigu est associé un mot cible lié à son acception dominante et un autre lié à son acception secondaire. Le mot amorce est soit l'item ambigu, soit une amorce neutre. Avec un SOA égal à 60 millisecondes (ms), seuls les temps de décision lexicale sur le mot relié à la signification fréquente des homographes fortement polarisés sont plus courts lorsque l'amorce est l'item ambigu que lorsqu'elle est neutre. Avec un SOA égal à $120 \mathrm{~ms}$, les deux acceptions des items ambigus faiblement et fortement polarisés sont activées, cette activation ne persistant que pour l'acception la plus fréquente avec un SOA égal à $200 \mathrm{~ms}$. Ces résultats apportent plusieurs informations. Premièrement, les deux acceptions d'un item ambigu peuvent être activées simultanément. Deuxièmement, la fréquence relative des acceptions constitue un facteur important dans le traitement des homographes (voir également Marquer, Le Nestour, Léveillé et Welitz, 1982 ; Mullet, 1994; Simpson, 1981 ; Tabossi, 1988). Enfin, si les deux acceptions sont disponibles de façon temporaire, seule l'acception dominante demeure activée après un certain temps. La désactivation de l'acception secondaire peut intervenir lors d'une étape tardive de l'accès à la signification du mot, durant laquelle les ressources attentionnelles de capacité limitée sont attribuées à l'acception la plus activée (Posner et Snyder, 1975). Une explication alternative réside dans l'hypothèse d'une relation inhibitrice entre les deux acceptions, en supposant que la compétition se développe avec le temps.

L'activation simultanée des différentes acceptions est compatible avec l'hypothèse d'une entrée lexicale unique sans com- 
pétition entre les deux acceptions. En revanche, l'hypothèse des entrées lexicales multiples s'accommode difficilement de cet accès exhaustif. En effet, la compétition entre les entrées lexicales a pour conséquence qu'une seule entrée parvient à être activée, en l'occurrence la plus fréquente ou celle qui s'avère contextuellement appropriée. De même, la compétition entre les acceptions envisagée conjointement à l'hypothèse d'une entrée lexicale unique empêche que les deux acceptions soient pleinement activées en même temps après la présentation d'un item ambigu. Cependant, il se peut que l'activation provenant de l'entrée lexicale commune soit suffisante pour que les deux acceptions atteignent un seuil d'activation pour être détectées. Ainsi, si l'hypothèse des entrées lexicales multiples n'est pas compatible avec l'accès exhaustif aux différentes acceptions, ce résultat ne permet pas de départager clairement les hypothèses de la présence ou de l'absence d'une compétition entre les acceptions envisagées conjointement à une entrée lexicale unique. Pour y parvenir, il est possible d'utiliser les effets de contexte, non pas dans le cadre d'une confrontation entre les conceptions modulariste et interactive, mais pour déterminer si l'augmentation de l'activation de l'acception induite par le contexte entraîne la désactivation de l'autre acception.

\section{L'EFFET DU CONTEXTE LEXICAL}

L'étude de l'effet du contexte discursif sur l'accès à la signification permet de tester des hypothèses relatives à l'organisation de la représentation des homographes. Il est nécessaire d'un point de vue théorique et méthodologique de dissocier l'effet du contexte textuel ou phrastique, attribué à la représentation intégrée d'un texte ou d'une phrase, de l'effet du contexte lexical engendré par les relations sémantiques qu'entretiennent les mots formant le contexte avec les différentes acceptions (voir Forster, 1981). En utilisant un contexte textuel, l'interprétation des résultats est subordonnée au type d'architecture impliquée dans le traitement du langage. En revanche, l'interprétation de l'effet d'un contexte lexical simple - c'est-à-dire formé d'un seul mot n'est pas soumise à cette incertitude dans la mesure où les conceptions modulariste et interactive s'accordent à situer cet effet lors de la phase d'accès à la signification (Forster, 1989). De 
plus, les caractéristiques formelles et sémantiques d'un contexte formé d'un mot peuvent être aisément identifiées et contrôlées.

La première étude montrant clairement des effets de contexte de nature lexicale en l'absence de contexte phrastique sur l'accès à la signification des homographes a été présentée par Schvaneveldt, Meyer et Becker (1976). Trois mots sont successivement présentés à l'écran : le troisième mot, toujours relié à une acception d'un item ambigu, est précédé de ce dernier ou d'un mot non relié. Le premier mot est, soit relié à l'une ou l'autre des deux acceptions de l'item ambigu, soit non relié. Les résultats ont montré que seule l'acception contextuellement appropriée est activée après la présentation de l'item ambigu. Cependant, les sujets effectuent une décision lexicale sur chacun des trois mots, ce qui conduit à une durée séparant le début de la présentation du second mot du début de la présentation du troisième mot (SOA) d'environ 500 ms (Glucksberg, Kreuz et Rho, 1986). La conclusion d'un effet immédiat du contexte lexical sur l'accès à la signification des homographes ne peut donc être définitive. Mullet (1994) a également utilisé des triplets de mots présentés à la cadence de $300 \mathrm{~ms}$ par mot. En utilisant des mots homographes fortement polarisés, les temps de décision lexicale sur les mots reliés à l'acception dominante sont plus courts que ceux des mots reliés à l'acception secondaire et des mots non reliés lorsque le contexte formé d'un mot induit l'acception dominante, alors que l'effet d'amorçage est obtenu pour les mots cibles reliés aux deux acceptions lorsque le contexte induit l'acception secondaire. En revanche, lorsque les mots homographes sont faiblement polarisés, les deux acceptions sont activées quel que soit le contexte. Ces résultats sont conformes à l'hypothèse d'une interaction entre le degré de dominance d'une acception par rapport à l'autre et l'effet du contexte. Néanmoins, le caractère précoce de cette interaction n'est pas établi compte tenu de la durée de présentation des mots égale à $300 \mathrm{~ms}$. De plus, l'interprétation de ces résultats est problématique, dans la mesure où elle s'appuie sur des comparaisons entre des temps de décision sur des mots cibles différents.

Dans les expériences de Balota et Paul (1996), trois mots étaient successivement présentés à la cadence de $133 \mathrm{~ms}$ par mot et les sujets effectuaient une tâche de décision lexicale ou de lecture à voix haute sur le troisième mot. Lorsque le mot cible était un homographe, il pouvait être précédé de deux mots reliés cha- 
cun à une acception différente ( « fleur - idée - pensée »), d'un mot relié placé en première position ( « fleur - volet - pensée ») ou en seconde position ( « fioul - idée - pensée »), ou de deux mots non reliés ( « fioul - volet - pensée »). Conformément à l'hypothèse d'une compétition entre les deux acceptions, Balota et Paul ont prédit un patron sous-additif des effets d'amorçage qui correspond à une différence entre, d'une part, l'effet d'amorçage obtenu lorsque les deux mots amorces sont reliés et, d'autre part, la sommation des effets d'amorçage obtenus dans la condition dans laquelle seul le premier mot est relié et celle dans laquelle seul le second mot est relié. Les résultats ont montré un patron additif pour les mots homographes, et ceci avec la tâche de décision lexicale et la tâche de lecture à voix haute. Ainsi, les résultats ne permettent pas de conclure à l'existence d'une relation inhibitrice entre les acceptions des homographes.

Le travail que nous présentons a pour principal objectif de tester les différentes hypothèses envisagées sur la représentation des homographes en étudiant les effets d'un contexte lexical simple, en l'occurrence un mot, sur l'accès à la signification des homographes. Les homographes utilisés étaient nettement polarisés afin de déterminer si la fréquence relative des acceptions est susceptible de moduler l'effet du contexte lors de l'accès à la signification (Mullet, 1994; Simpson, 1981 ; Tabossi, 1988). Deux expériences de décision lexicale ont été réalisées, dans lesquelles les essais étaient constitués par la présentation successive d'un mot contexte dont la nature était manipulée, d'un item ambigu utilisé comme mot amorce, et d'un mot cible. Le mot cible était relié à l'acception dominante de l'item ambigu dans l'expérience 1 et à l'acception secondaire dans l'expérience 2.

L'effet du contexte lexical sur l'accès à la signification est par nature temporaire et diminue avec le temps. Parallèlement au déclin de cet effet, les sujets développent des stratégies afin d'effectuer la tâche demandée lorsqu'ils disposent du temps nécessaire pour le faire (Neely, 1991). Le second objectif de ces expériences est de déterminer les paramètres temporels optimums pour étudier l'effet du contexte lexical et, si possible, d'en dissocier la composante automatique de la composante stratégique. Si l'effet du contexte s'exerce avec les paramètres temporels les plus courts, celui-ci pourra être attribué aux processus intervenant lors de l'accès à la signification. En revanche, s'il ne se manifeste que de façon tardive - c'est-à-dire avec un SOA supé- 
rieur à $250 \mathrm{~ms}$ (Neely, 1991) - il sera imputé aux stratégies postérieures à l'accès au lexique. L'étude du décours temporel nous permettra également de déterminer si la compétition entre les acceptions nécessite du temps pour se développer. Aussi, le délai temporel séparant la présentation du mot amorce de celle du mot cible a été systématiquement manipulé (ISI : 50 ou $350 \mathrm{~ms}$ ), ainsi que le temps de présentation des mots contextes et amorces (100, 200 , ou $350 \mathrm{~ms}$ ). La majorité des études concernant l'effet du contexte phrastique sur l'accès à la signification des homographes ont utilisé une présentation auditive des stimulus, alors que celles étudiant l'accès à la signification en l'absence de contexte ont utilisé une présentation uni-modale. Une présentation visuelle des stimulus a été adoptée afin que le temps de présentation des mots contextes et amorces ne dépende pas du nombre de lettres qui les composent. Une troisième expérience a été réalisée afin de déterminer la source de l'effet du contexte constaté dans les deux premières expériences. Plus précisément, il s'agit de vérifier que les effets obtenus étaient imputables à l'effet du mot contexte sur l'accès à la signification de l'item ambigu, et non pas à la relation directement entretenue par les mots contextes et cibles reliés à la même acception de l'item ambigu.

HYPOTHËSES ET PRÉDICTIONS

\section{Effet du contexte lexical approprié}

Le matériel expérimental a été élaboré afin que le mot contexte puisse faciliter l'accès à la signification à laquelle il est relié (Hla). Ainsi, nous observerons des temps de décision plus courts lorsque le mot contexte et le mot cible sont reliés à la même acception de l'item ambigu présenté en tant que mot amorce que lorsque le mot contexte n'est relié à aucune des deux acceptions, et ceci dès le temps de présentation égal à $100 \mathrm{~ms}$ et l'ISI égal à $50 \mathrm{~ms}$. En revanche, si le contexte lexical n'exerce pas de contrainte suffisante sur l'accès à la signification des homographes $(\mathrm{H} 1 b)$, alors l'effet du contexte approprié ne se manifestera que de façon tardive, lorsque les sujets bénéficieront du temps suffisant pour utiliser des stratégies postérieures à l'accès au lexique. Dans ce cas, les expériences ne permettront pas de tester l'hypothèse d'une compétition entre les deux acceptions. 


\title{
Effet du contexte non approprié
}

Selon l'hypothèse d'une compétition entre les entrées lexicales (Forster, 1976 ; Kellas et al., 1988) ou entre les acceptions (Gottlob et al., 1999; Kintsch, 1988), l'augmentation de l'activation d'une acception par le contexte entraîne la diminution de l'activation de l'autre acception (H2a). Aussi, les temps de décision lexicale seront plus longs lorsque le mot contexte et le mot cible sont reliés à une acception différente de l'item ambigu que lorsque le mot contexte n'est relié à aucune des deux acceptions, et ceci dès le temps de présentation égal à $100 \mathrm{~ms}$ et l'ISI égal à $50 \mathrm{~ms}$. À l'inverse, selon l'hypothèse d'une entrée lexicale unique sans compétition entre les deux acceptions, nous observerons une absence de différence des temps de décision entre la condition dans laquelle le mot contexte et le mot cible sont reliés à une acception différente et celle dans laquelle le mot contexte est non relié $(\mathrm{H} 2 b)$.

\section{EXPÉRIENCE 1: MOT CIBLE RELIÉ À L'ACCEPTION DOMINANTE}

\author{
MÉTHODE
}

MATÉRIEL

Le matériel expérimental était constitué de 24 triplets de mots sélectionnés à l'aide de deux épreuves préliminaires (Thérouanne, 2000). La première avait pour objectif d'évaluer la fréquence relative des acceptions des homographes. Pour chacun des mots homographes présentés, 72 étudiants avaient pour consigne d'écrire le plus spontanément possible un mot ou un groupe de mots correspondant au premier sens de l'item ambigu qui leur venait à l'esprit. À l'issue du regroupement des réponses selon des critères sémantiques, seuls les mots homographes suscitant des réponses correspondant à deux significations clairement distinctes ont été retenus. De plus, la fréquence d'occurrence des réponses correspondant à la signification dominante devait être comprise entre 70 et $90 \%$.

La seconde épreuve avait pour but de sélectionner les mots utilisés ultérieurement en tant que mots contextes et cibles. À chacun des mots homographes sélectionnés à l'issue de la première épreuve étaient associés un mot inducteur correspondant à l'acception dominante et un mot induc- 
teur correspondant à l'acception secondaire'. Ces mots inducteurs exprimaient la catégorie sémantique à laquelle appartenait l'acception. Les 90 étudiants participant à l'épreuve devaient écrire le plus rapidement et le plus spontanément possible le premier mot qui leur venait à l'esprit après la lecture de chaque couple de mots présentés. À partir des réponses ainsi produites, nous avons sélectionné 24 mots homographes pour lesquels la présence des deux acceptions était confirmée. À chacun de ces items ambigus ont été affectés deux mots cibles utilisés dans les expériences présentées par la suite. Les mots cibles choisis étaient ceux qui possédaient la fréquence d'occurrence la plus élevée dans les normes. Par exemple, pour l'item ambigu « palais », les mots « château »et « goût » ont été choisis pour être respectivement utilisés en tant que mots cibles dominant (expérience 1) et secondaire (expérience 2). Par ailleurs, trois mots appelés par la suite « mots contextes » étaient affectés à chaque item ambigu : le premier était relié à l'acception dominante, le second relié à l'acception secondaire, et le troisième n'était sémantiquement relié à aucune des deux acceptions. Les mots contextes dominant et secondaire ont été sélectionnés afin que leur fréquence moyenne d'occurrence dans les normes issues de la seconde épreuve soit comparable. Le nombre de lettres, le point d'unicité orthographique et le nombre de voisins orthographiques dans la langue des mots contextes ont été contrôlés à l'aide de la base de données BRLiLEX (Content, Mousty et Radeau, 1990) afin que les valeurs moyennes de ces trois caractéristiques lexicales ne diffèrent pas en fonction des conditions expérimentales. Le matériel expérimental utilisé dans les expériences 1 et 2 est présenté en annexe et un exemple est donné dans le tableau $I$.

\section{Tableauj I. - Exemple de matériel expérimental utilisé dans les expériences 1 et 2}

Example of experimental stimuli used in experiments 1 and 2

\begin{tabular}{|c|c|c|c|c|c|}
\hline \multirow[b]{2}{*}{ Expérience } & \multicolumn{3}{|c|}{ Mot contexte } & \multirow[b]{2}{*}{ Mot amorce } & \multirow[b]{2}{*}{ Mot cible } \\
\hline & dominant & secondaire & non relié & & \\
\hline expérience 1 & princesse & mâchoire & brin & palais & château \\
\hline expérience 2 & princesse & mâchoire & brin & palais & goût \\
\hline
\end{tabular}

1. Le mot inducteur a été utilisé afin d'obtenir des mots contextes et cibles reliés à l'acception secondaire done les fréquences d'occurrence des réponses sont comparables à celles des mots contextes et cible reliés à l'acception dominante. En effet, si l'on utilise une épreuve d'association libre, les réponses ainsi produites sont en grande majorité reliées à l'acception dominante de l'item ambigu ('Tabossi, 1988). 
Le matériel de remplissage comportait 40 triplets de mots afin d'obtenir dans la phase expérimentale (1) autant de cibles constituées de mots que de pseudo-mots, (2) autant de cibles sémantiquement reliées à l'amorce que de cibles non reliées, (3) autant de mots amorces ambigus que de mots amorces non ambigus, et (4) autant de mots contextes sémantiquement reliés au mot amorce que de mots contextes non reliés.

La phase expérimentale était divisée en deux blocs de 32 essais. Dans chaque bloc, l'ordre de présentation des essais était tiré aléatoirement pour chaque sujet. Six listes de matériel ont été construites de manière à ce que chaque item expérimental apparaisse dans chaque condition et dans chaque bloc et ne soit vu qu'une seule fois par chaque sujet.

\section{PROCÉDCRE EXPÉRIMENTALE}

Les sujets étaient d'abord entraînés à la tâche de décision lexicale lors d'une phase de familiarisation qui comportait 96 essais constitués par la présentation successive d'un mot amorce et d'un mot cible. Les sujets étaient ensuite confrontés à la phase expérimentale proprement dite, comprenant 8 essais d'entraînement et deux blocs de 32 essais expérimentaux. Les consignes étaient administrées par écrit et l'expérimentateur insistait oralement sur la nécessité de concilier la rapidité et l'exactitude lors de la tâche de décision lexicale. L'expérience, pilotée par un ordinateur Macintosh, se déroulait individuellement et durait environ quarante minutes.

Un essai de la phase d'entraînement et de la phase expérimentale comportait la séquence suivante. Un cercle noir apparaissait au centre de l'écran pendant une seconde pour signaler le début de l'essai. Le mot contexte était ensuite présenté pendant $100 \mathrm{~ms}, 200 \mathrm{~ms}$, ou $350 \mathrm{~ms}$ en fonction de la condition expérimentale. Après un intervalle de $50 \mathrm{~ms}$, le mot amorce était présenté pendant la même durée que celle du mot contexte. Puis, l'item ambigu était remplacé par six cercles noirs constituant un masque présenté pendant 50 ou $350 \mathrm{~ms}$ selon la condition expérimentale. Après la disparition des cercles, une suite de lettres était présentée à l'écran. Les sujets devaient décider si cette suite de lettres constituait ou non un mot de la langue française. La touche « = » du clavier correspondait à la réponse « OUI » et la touche « w » à la réponse « NON » pour les sujets droitiers et inversement pour les sujets gauchers. Après chaque essai, un message indiquant l'exactitude et le temps de la réponse fournie apparaissait à l'écran pendant une seconde. Les sujets commandaient eux-mêmes le début de l'essai suivant en appuyant sur la barre « espace » du clavier.

À la fin de chaque bloc d'essais, les sujets réalisaient une épreuve de reconnaissance afin de s'assurer qu'ils avaient lu les mots constituant chaque essai. Les sujets devaient décider pour chacun des 32 couples de mots inscrits sur une feuille s'ils étaient apparus dans le bloc d'essais auquel ils venaient d'être confrontés. La moitié des couples présentés étaient effectivement apparus lors du bloc d'essai considéré. 
SIJETS

Cent huit étudiants de langue maternelle française ont participé à l'expérience. Ies sujets suivaient une formation de l'enseignement supérieur à l'Université Aix-Marseille I. Trois sujets étaient aléatoirement affectés à chacune des six listes de matériel expérimental, et ceci pour chaque modalité des facteurs Temps de présentation et ISI. Douze sujets supplémentaires issus de la même population ont participé à l'expérience afin de remplacer les sujets dont les protocoles individuels comportaient, soit plus de 12 erreurs de décision lexicale pour un total de 64 essais, soit plus de $35 \%$ d'erreurs à l'épreuve de reconnaissance.

\section{RÉST:ITATS}

Les temps correspondant aux erreurs de décision lexicale étaient éliminés $(3,4 \%$ des essais expérimentaux), ainsi que les temps de décision supérieurs à la moyenne plus trois écarts types ou inférieurs à la moyenne moins trois écarts types considérés comme des valeurs aberrantes $(1,4 \%)$. Les moyennes des temps de réponse et des pourcentages d'erreurs de décision lexicale sont présentées dans le tableau II.

Deux analyses ont été effectuées sur deux variables dépendantes, le temps moyen et le pourcentage d'erreurs de décision lexicale selon les plans suivants : $S 18<\mathrm{T} 3 * \mathrm{~V} 2>{ }^{*} \mathrm{C} 3$ et $I 24{ }^{*} \mathrm{~T} 3{ }^{*} \mathrm{~V} 2{ }^{*} \mathrm{C} 3$, les lettres $S, I, \mathrm{~T}, \mathrm{~V}$ et $\mathrm{C}$ renvoyant respectivement aux facteurs Sujets (facteur aléatoire), Items (facteur aléatoire), Temps de présentation $(100,200$ ou $350 \mathrm{~ms})$, Intervalle inter-stimulus (50 ms ou $350 \mathrm{~ms}$ ) et Contexte (dominant, secondaire, ou non relié). L'ensemble des analyses a été réalisé à l'aide du logiciel StatViewß. Selon l'usage, «F1》 et « $t 1$ »renvoient aux analyses prenant le facteur Sujets comme source de variation aléatoire, alors que « $F 2$ »et « $t 2$ » renvoient aux analyses par items. Le seuil de signification est fixé à $5 \%$ de risque de rejeter à tort l'hypothèse nulle.

Le facteur Contexte exerce un effet significatif sur le temps moyen de décision lexicale $(F 1(2,204)=13,05, p<.001$; $F 2(2,46)=8,23, p<.001):$ les temps de décision sont plus courts lorsque le mot contexte est relié à l'acception dominante que lorsqu'il est non relié $(d=22 \mathrm{~ms}, \quad F 1(1,204)=17,18$, $p<.001 ; \mathrm{F} 2(1,46)=10,68, p<.01)$, alors que la seconde comparaison planifiée ne montre pas de différence entre les condi- 
Tableau II. - Temps de décision lexicale (en ms), écart type (entre parenthèses) et pourcentage d'erreurs en fonction du temps de présentation, de l'ISI et du mot contexte. Expérience 1

Mean lexical decision latencies (in ms), standard deviations (in parentheses), and error percentage as a function of Presentation time, Context word and ISI factors. Experiment 1

\begin{tabular}{llccc} 
& & \multicolumn{3}{c}{ Mot contexte } \\
\cline { 3 - 5 } Présentation & ISI & dominant & non relié & secondaire \\
\hline \multirow{3}{*}{$100 \mathrm{~ms}$} & \multirow{3}{*}{$50 \mathrm{~ms}$} & $556(98)$ & $588(106)$ & $567(94)$ \\
& & $4,9 \%$ & $5,6 \%$ & $5,6 \%$ \\
& \multirow{3}{*}{$350 \mathrm{~ms}$} & $500(53)$ & $514(88)$ & $522(73)$ \\
& \multirow{3}{*}{$200 \mathrm{~ms}$} & $4,9 \%$ & $2,1 \%$ & $4,9 \%$ \\
\hline \multirow{3}{*}{$50 \mathrm{~ms}$} & $491(43)$ & $515(56)$ & $507(40)$ \\
& \multirow{3}{*}{$350 \mathrm{~ms}$} & $1,4 \%$ & $3,5 \%$ & $2,1 \%$ \\
& \multirow{3}{*}{$50 \mathrm{~ms}$} & $500(93)$ & $536(105)$ & $536(107)$ \\
& & $1,4 \%$ & $3,5 \%$ & $4,9 \%$ \\
\hline \multirow{3}{*}{$350 \mathrm{~ms}$} & $534(84)$ & $542(76)$ & $578(81)$ \\
& & $1,4 \%$ & $1,4 \%$ & $1,4 \%$ \\
& \multirow{2}{*}{ Moyenne } & $518(65)$ & $542(113)$ & $542(69)$ \\
& & $3,5 \%$ & $3,5 \%$ & $6,3 \%$ \\
\hline
\end{tabular}

tions « secondaire» et « non relié » $\left(d=-3 \mathrm{~ms}, \mathrm{~F}^{\prime} s<1\right)$. L'interaction entre les facteurs Contexte et Temps de présentation n'est pas significative $(\mathrm{F} 1(4,204)=1,14$, n.s. ; F2 $<1)$, tout comme celle entre les facteurs Contexte et ISI $\left(\mathrm{F}^{\prime} s<1\right)$. L'interaction impliquant les facteurs Contexte, Temps de présentation et ISI est également non significative $(F 1(4,204)=1,79$, n.s. ; $F 2(4,92)=1,31$, n.s. $)$.

L'effet du facteur Contexte sur le pourcentage d'erreurs n'est pas significatif $(\mathrm{Fl}(2,204)=2,17$, n.s. ; F2 $(2,46)=2,71$, n.s. $)$. Le pourcentage d'erreurs ne diffère pas entre les conditions « dominant » et « non relié » $(F 1<1 ; F 2<1)$. En revanche, le pourcentage d'erreurs est plus important dans la condition « secondaire » que dans la condition « non relié », bien que 
cette différence n'atteigne pas le seuil de signification $(F 1(1,204)=2,76$, n.s. ; F2 $(1,46)=3,73$, n.s. $)$. L'interaction entre les facteurs Contexte et Temps de présentation, celle entre les facteurs Contexte et ISI, et l'interaction impliquant les trois facteurs manipulés ne sont pas significatives $\left(\mathbf{F}^{\prime} s<1\right)$. Ainsi, il n'y a pas dans cette expérience de comportement compensatoire entre la rapidité et l'exactitude de la réponse.

Bien que le facteur Contexte n'interagisse ni avec le Temps de présentation, ni avec l'ISI, une lecture détaillée des résultats révèle que le contexte exerce un effet différent selon les modalités des deux facteurs temporels. Aussi, nous avons réalisé des analyses testant l'effet du contexte approprié au mot cible (dominant) et non approprié (secondaire) sur les temps de décision lexicale pour chacune des six conditions formées par le croisement des deux facteurs temporels. Les résultats de ces analyses sont présentés dans le tableau III.

Tableau III. - Effet du mot contexte relié à l'acception dominante et de celui relié à l'acception secondaire sur les temps de décision lexicale du mot cible relié à l'acception dominante. Expérience 1

Effect of dominant and subordinate context words on lexical decision latencies. Experiment 1

\begin{tabular}{|c|c|c|c|c|c|c|c|}
\hline \multirow[b]{2}{*}{$\begin{array}{l}\text { Temps de } \\
\text { présentation }\end{array}$} & \multirow[b]{2}{*}{ ISI (SOA) } & \multicolumn{3}{|c|}{ Mot contexte dominant } & \multicolumn{3}{|c|}{ Mot contexte secondaire } \\
\hline & & $\begin{array}{l}\text { effet du } \\
\text { contexte }\end{array}$ & $\mathrm{t} 1(17)$ & $\mathrm{t} 2(23)$ & $\begin{array}{l}\text { effet du } \\
\text { contexte }\end{array}$ & t1(17) & t2(23) \\
\hline \multirow[t]{2}{*}{100} & $50(150)$ & +32 & $2,17^{*}$ & $2,93^{* *}$ & +21 & 1,26 & 1,02 \\
\hline & $350(450)$ & +14 & 1,19 & $<1$ & -8 & $<1$ & $<1$ \\
\hline \multirow[t]{2}{*}{200} & $50(250)$ & +23 & 1,51 & $2,14^{*}$ & +8 & $<1$ & $<1$ \\
\hline & $350(550)$ & +36 & $2,49^{*}$ & $2,07^{*}$ & 0 & $<1$ & $<1$ \\
\hline \multirow[t]{2}{*}{350} & $50(400)$ & +8 & $<1$ & $<1$ & -36 & $2,81^{*}$ & $3,77^{* *}$ \\
\hline & $350(700)$ & +24 & 1,23 & 1,07 & -2 & $<1$ & $<1$ \\
\hline
\end{tabular}

Notes. (1) L'effet du contexte correspond à la différence entre le temps moyen de décision lexicale dans la condition dominant ou secondaire et le temps de décision dans la condition non relié.

(2) $*: p<.01, *: p<.05$. 
Pour le son le plus court, les temps de décision lexicale sur le mot cible relié à l'acception dominante sont plus courts lorsque le mot contexte est relié à cette même acception que lorsqu'il est non relié. Cet effet du contexte persiste lorsque le SOA est égal à $250 \mathrm{~ms}$, bien que la différence ne soit significative que dans l'analyse par item. Ainsi, conformément à l'hypothèse Hla, le contexte lexical exerce des contraintes suffisantes pour faciliter de façon précoce l'accès à la signification à laquelle il est relié. L'effet du contexte approprié semble disparaître avec un SOA égal à $400 \mathrm{~ms}$, montrant la nature transitoire de l'effet attribué au mot contexte. Pour les sOA plus longs, l'effet du contexte approprié n'est obtenu qu'avec le $S O A$ égal à $550 \mathrm{~ms}$. Le fait que l'effet du contexte disparaît, puis réapparaît de façon transitoire semble montrer que sa manifestation tardive est imputable à des processus postérieurs à l'accès lexical qui se développent lorsque les sujets disposent du temps nécessaire (voir Neely, 1991), qu'ils soient de nature consciente ou non.

Par ailleurs, une inhibition relative des temps de réponse est observée lorsque le contexte est relié à l'acception secondaire de l'item ambigu. Cependant, cet effet d'inhibition n'intervient que de manière tardive lorsque le SOA est égal à $400 \mathrm{~ms}$ et n'est pas obtenu conjointement à un effet du contexte lexical approprié. Ainsi, cet effet ne reflète pas une diminution de l'activation de l'acception secondaire du fait d'une quantité d'inhibition supplémentaire engendrée par l'augmentation de l'activation de l'acception dominante. L'absence de différence, entre la condition dans laquelle le mot contexte est relié à une acception différente de celle du mot cible et celle dans laquelle le mot contexte est non relié pour les SOA les plus courts, est compatible avec l'hypothèse selon laquelle il n'existe pas de compétition entre les représentations des différentes acceptions $(\mathrm{H} 2 b)$.

\section{EXPÉRIENCE 2 : MOT CIBLE RELIÉ À L'ACCEPTION SECONDAIRE}

\section{MATÉRIEL ET PROCÉdURE EXPÉRIMENTALE}

Le matériel utilisé dans la seconde expérience reprenait celui de la première. La seule différence résidait dans la nature du mot cible utilisé qui était toujours relié à l'acception secondaire de l'item ambigu présenté en 
tant que mot amorce (par exemple, « goût » pour l'item ambigu « palais »). Dès lors, la condition expérimentale dans laquelle le mot contexte est approprié au mot cible est la condition « secondaire », et non pas « dominant » comme ce fut le cas précédemment. La procédure expérimentale et le plan d'expérience demeuraient en tout point identiques à ceux de l'expérience $\mathrm{I}$.

SI:JETS

Cent huit étudiants de langue maternelle française ont participé à l'expérience. Les sujets suivaient tous une formation de l'enseignement supérieur à l'Université Aix-Marseille I. Comme dans la précédente expérience, trois sujets étaient affectés aléatoirement à chacune des six listes de matériel expérimental, et ceci pour chaque modalité du facteur Temps de présentation et du facteur Isi. Quinze sujets supplémentaires ont participé à l'expérience afin de remplacer les sujets dont les protocoles individuels comportaient, soit plus de 12 erreurs de décision lexicale pour un total de 64 essais, soit plus de $35 \%$ d'erreurs à l'épreuve de reconnaissance.

RÉSLITTATS

Comme ce fut le cas dans la première expérience, les temps correspondant aux erreurs de décision lexicale étaient éliminés $(3,0 \%)$, ainsi que les temps de décision supérieurs à la moyenne plus trois écarts types ou inférieurs à la moyenne moins trois écarts types $(1,6 \%)$. Deux analyses de la variance ont été effectuées sur le temps moyen et le pourcentage d'erreurs de décision lexicale selon les plans d'analyse déjà utilisés dans la première expérience. Les moyennes des temps de décision lexicale sont présentées dans le tableau IV.

L'effet du facteur Contexte sur le temps de décision lexicale est significatif $(F(2,204)=16,80, p<.001 ; F 2(2,46)=14,95$, $p<.001)$. Le temps moyen de décision est plus court lorsque le mot contexte est relié à l'acception secondaire que lorsqu'il est non relié $(\mathrm{F} 1(1,204)=28,36, p<.001 ; \quad F 2(1,46)=22,28$, $p<.001$ ), alors que la seconde comparaison ne montre pas de différence entre les conditions «dominant 》 et «non relié » $(F 1<1 ; F 2<1)$. L'interaction entre les facteurs Contexte et Temps de présentation, celle entre les facteurs Contexte et ISI et celle impliquant les trois facteurs manipulés ne sont pas significatives $\left(F^{\prime} s<1\right)$. 
Tableau IV. - Temps de décision lexicale (en ms), écart type (entre parenthèses) et pourcentage d'erreurs en fonction du temps de présentation, de l'ISI et du mot contexte. Expérience 2

Mean lexical decision latencies (in ms), standard deviations (in parentheses), and error percentage as a function of Presentation time, Context word and ISI factors. Experiment 2

\begin{tabular}{|c|c|c|c|c|}
\hline \multirow[b]{2}{*}{ Présentation } & \multirow[b]{2}{*}{ ISI } & \multicolumn{3}{|c|}{ Mot contexte } \\
\hline & & dominant & non relié & secondaire \\
\hline \multirow{4}{*}{$100 \mathrm{~ms}$} & \multirow{2}{*}{$50 \mathrm{~ms}$} & $580(93)$ & 593 (99) & $553(86)$ \\
\hline & & $3,5 \%$ & $2,8 \%$ & $4,9 \%$ \\
\hline & \multirow{2}{*}{$350 \mathrm{~ms}$} & $530(83)$ & $533(69)$ & $495(59)$ \\
\hline & & $4,9 \%$ & $0,7 \%$ & $1,4 \%$ \\
\hline \multirow{4}{*}{$200 \mathrm{~ms}$} & \multirow{2}{*}{$50 \mathrm{~ms}$} & $532(97)$ & $544(96)$ & $510(75)$ \\
\hline & & $3,5 \%$ & $2,1 \%$ & $1,4 \%$ \\
\hline & \multirow{2}{*}{$350 \mathrm{~ms}$} & $534(77)$ & $535(77)$ & $523(66)$ \\
\hline & & $2,1 \%$ & $0,7 \%$ & $0,7 \%$ \\
\hline \multirow{5}{*}{$350 \mathrm{~ms}$} & \multirow{2}{*}{$50 \mathrm{~ms}$} & $548(60)$ & $541(70)$ & $527(61)$ \\
\hline & & $5,6 \%$ & $4,9 \%$ & $2,8 \%$ \\
\hline & \multirow{2}{*}{$350 \mathrm{~ms}$} & $531(73)$ & $530(101)$ & $500(60)$ \\
\hline & & $5,6 \%$ & $1,4 \%$ & $3,5 \%$ \\
\hline & Moyenne & $543(4,2)$ & $546(2,1)$ & $518(2,4)$ \\
\hline
\end{tabular}

L'effet du Contexte sur le pourcentage d'erreurs est significatif dans l'analyse par sujets $(F 1(2,204)=4,32, p<.05)$, mais n'atteint pas le seuil de signification dans l'analyse par items $(F 2(2,46)=3,03$, n.s. $):$ le pourcentage d'erreurs ne diffère pas entre les conditions « secondaire » et «non relié » $(F 1<1$; F2 $<1$ ). En revanche, le pourcentage d'erreurs est plus important dans la condition « dominant » que dans la condition « non relié » $(\mathrm{F} 1(1,204)=7,51, p<.01 ; \mathrm{F} 2(1,46)=5,27, p<.05)$. L'interaction entre les facteurs Contexte et Temps de présentation n'est pas significative $(F 1<1 ; F 2<1)$, de même que celle entre les facteurs Contexte et ISI $(F 1(2,204)=1,16$, n.s. ; $F 2(2,46)=1,60$, n.s.). L'interaction impliquant les facteurs 
Contexte, Temps de présentation et ISI se révèle également non significative $(\mathrm{F} 1(4,204)=1,07$, n.s. ; F2 $<1)$.

Comme ce fut le cas dans la première expérience, des analyses ont testé l'effet du contexte dans chacune des six conditions formées par le croisement des facteurs Temps de présentation et ISI. Les résultats de ces analyses sont présentés dans le tableau V.

Tableat: V. - Effet du mot contexte relié à l'acception dominante et de celui relié à l'acception secondaire sur les temps de décision lexicale du mot cible relié à l'acception secondaire. Expérience 2

Effect of dominant and subordinate context words on lexical decision latencies. Experiment 2

\begin{tabular}{llcccccccc} 
& & \multicolumn{3}{c}{ Mot cible dominant } & & \multicolumn{3}{c}{ Mot cible secondaire } \\
\cline { 3 - 4 } $\begin{array}{l}\text { Temps de } \\
\text { présentation }\end{array}$ & ISI (SOA) & $\begin{array}{c}\text { effet du } \\
\text { contexte }\end{array}$ & t1(17) & t2(23) & & $\begin{array}{c}\text { effet du } \\
\text { contexte }\end{array}$ & t1(17) & t2(23) \\
\hline 100 & $50(150)$ & +13 & $<1$ & $<1$ & & +40 & $2,90^{* *}$ & $2,81^{* *}$ \\
& $350(450)$ & +3 & $<1$ & $<1$ & & +38 & $4,17^{* *}$ & $4,14^{* *}$ \\
200 & $50(250)$ & +12 & $<1$ & $<1$ & & +34 & $3,48^{* *}$ & 2,25 \\
& $350(550)$ & +1 & $<1$ & $<1$ & & +11 & 1,23 & $<1$ \\
350 & $50(400)$ & -7 & $<1$ & $<1$ & & +13 & $<1$ & $<1$ \\
& $350(700)$ & -1 & $<1$ & $<1$ & & +30 & 1,80 & $3,49^{* *}$ \\
\hline
\end{tabular}

Note. ${ }^{* *}: p<.01,{ }^{*}: p<.05$.

En considérant les SOA de 150 et $250 \mathrm{~ms}$, les résultats observés avec le mot cible relié à l'acception secondaire reproduisent ceux observés avec le mot cible relié à l'acception dominante : les temps de décision lexicale sont plus courts lorsque le mot contexte est relié à la même acception que le mot cible que lorsqu'il est non relié. Cet effet du contexte approprié est également obtenu pour les SOA de 450 et $700 \mathrm{~ms}$, mais pas pour les SOA de 400 et $550 \mathrm{~ms}$. Quel que soit le SOA, aucune différence n'est constatée entre la condition dans laquelle le mot contexte est relié à une acception différente du mot cible et celle dans laquelle il est non relié. Une différence entre ces deux conditions a néan- 
moins été obtenue dans les analyses portant sur le pourcentage d'erreurs. Cependant, cette différence n'atteignait le seuil de signification que dans les analyses par items et pour le SOA égal à $450 \mathrm{~ms}(t 1(17)=1,68, n . s . ; t 2(23)=2,03, p=.05)$ et celui égal à $700 \mathrm{~ms}(t 1(17)=1,84$, n.s. ; $t 2(23)=2,06, p=.05)$. Ainsi, l'ensemble des résultats est compatible avec l'hypothèse de l'absence de compétition entre les représentations relatives aux différentes acceptions.

Les résultats des deux premières expériences ont montré un effet d'amorçage lorsque les mots contextes et cibles étaient reliés à la même acception de l'item ambigu présenté en tant que mot amorce. Les résultats ont été interprétés en termes de contraintes contextuelles exercées par le mot contexte sur l'accès à la signification de l'item ambigu. Néanmoins, il existe une interprétation alternative de ce résultat selon laquelle l'effet du contexte est attribuable à un effet d'amorçage direct du mot contexte relié à une acception sur le mot cible relié à cette même acception. Si tel est le cas, l'effet du contexte persistera en l'absence de l'homographe présenté en tant que mot amorce. Une troisième expérience a donc été réalisée afin de déterminer si le mot contexte est susceptible d'exercer un effet direct sur le mot cible dans des conditions analogues à celles des deux premières expériences. Comme précédemment, les essais étaient constitués par la présentation successive de trois mots. Le principe de l'expérience était d'enregistrer les temps de décision lexicale sur un même mot cible alors que les mots contextes et amorces étaient manipulés. Le temps de présentation des mots contextes et cibles était fixé à $100 \mathrm{~ms}$, et l'ISI à 50 ms'.

\section{EXPÉRIENCE 3}

MATÉRIEL

Le matériel expérimental était constitué de 24 triplets de mots contexte-amorce-cible et reprenait les 24 items ambigus, ainsi que les mots

1. Un mot sémantiquement non relié au mot cible et utilisé comme mot amorce est susceptible d'entraîner un effet d'inhibition de nature sémantique (voir Masson, 1995). Cependant, ce dernier ne se manifeste pas lorsque le SOA est relativement court, c'est-à-dire inférieur à 360 ms (Balota et Paul, 1996 ; Lavigne-Tomps et Vitu, 1997). 
utilisés en tant que contexte et cible dans les expériences 1 et 2 . Le mot cible était, soit relié à l'acception dominante de l'item ambigu, soit à son acception secondaire. Le mot amorce était l'item ambigu ou un mot non ambigu qui n'était relié à aucune des deux acceptions. Le nombre de lettres de l'item ambigu et du mot non ambigu était identique. De plus, ces deux mots étaient appariés pour les caractéristiques lexicales suivantes à l'aide de la base de données BRLLEX : la fréquence d'usage dans la langue, le point d'unicité orthographique, le nombre de voisins orthographiques dans la langue, et la fréquence moyenne des digrammes. La nature du mot contexte était également manipulée : ce mot était relié à la même acception que le mot cible ou n'était relié à aucune des deux acceptions. Un exemple de matériel expérimental est présenté dans le tableau VI.

\section{TABILAC VI. - Exemple de matériel expérimental de l'expérience 3}

Example of experimental stimuli used in Experiment 3

\begin{tabular}{|c|c|c|c|c|c|c|c|c|}
\hline \multirow{3}{*}{$\begin{array}{l}\text { Amorce } \\
\text { Contexte }\end{array}$} & \multicolumn{4}{|c|}{ Mot cible relié à l'acception dominante } & \multicolumn{4}{|c|}{ Mot cible relié à l'acception secondaire } \\
\hline & \multicolumn{2}{|c|}{ homographe } & \multicolumn{2}{|c|}{ non reliée } & \multicolumn{2}{|c|}{ homographe } & \multicolumn{2}{|c|}{ non reliée } \\
\hline & relié & non relié & relié & non relié & relié & non relié & relié & non relié \\
\hline contexte & princesse & brin & princesse & brin & mâchoire & brin & mâchoire & brin \\
\hline amorce & palais & palais & preuve & preuve & palais & palais & preuve & preuve \\
\hline cible & château & château & château & château & goût & gout & gout & gout \\
\hline
\end{tabular}

Le matériel de remplissage comportait 24 triplets dont le stimulus cible était un pseudo-mot. L'ordre de présentation des essais était tiré aléatoirement pour chaque sujet. Huit conditions expérimentales étaient formées par le croisement des facteurs Fréquence relative du mot cible, Amorce et Contexte. Huit listes de matériel ont été construites de manière à ce que chaque item expérimental ne soit vu qu'une seule fois par chaque sujet et apparaisse dans toutes les conditions.

\section{PROCÉdjRE}

Les sujets étaient d'abord entraînés à la tâche de décision lexicale lors d'une phase de familiarisation qui comportait $\mathbf{4 8}$ essais constitués par la présentation successive d'un mot amorce et d'un mot cible. Les sujets étaient ensuite confrontés à la phase expérimentale proprement dite, comprenant 8 essais d'entraînement et 48 essais expérimentaux. Les consignes étaient administrées par oral et l'expérimentateur insistait sur la nécessité 
de concilier la rapidité et l'exactitude lors de la tâche de décision lexicale. L'expérience se déroulait individuellement et durait environ vingt minutes.

Un essai de la phase d'entraînement et de la phase expérimentale comportait la séquence d'événements suivante: un cercle apparaissait au centre de l'écran pendant une seconde pour signaler le début de l'essai. Le mot contexte était ensuite présenté pendant $100 \mathrm{~ms}$. Après un intervalle de $50 \mathrm{~ms}$, le mot amorce était présenté pendant $100 \mathrm{~ms}$ et était remplacé par une série de 10 dièses constituant un masque présenté pendant $50 \mathrm{~ms}$. Après la disparition des dièses, une suite de lettres était présentée à l'écran. Les sujets devaient décider si cette suite de lettres constituait ou non un mot de la langue française. Lors de la phase d'entraînement, un message qui indiquait l'exactitude de la réponse fournie ainsi que le temps de la réponse apparaissait à l'écran pendant une seconde. Ce message n'était pas fourni pendant la phase expérimentale. Les sujets commandaient euxmêmes le début de l'essai suivant en appuyant sur la barre « espace » du clavier.

À la fin de chaque bloc d'essais, les sujets réalisaient une épreuve de reconnaissance afin de s'assurer qu'ils avaient lu les mots constituant chaque essai. Les sujets devaient décider pour chacun des 16 couples de mots inscrits sur une feuille s'ils étaient apparus dans le bloc d'essais auquel ils venaient d'être confrontés. La moitié des couples étaient effectivement apparus pendant l'expérience.

\section{SUJETS}

Vingt-quatre étudiants de l'Université d'Aix-Marseille I ont participé à l'expérience. Les sujets étaient tous de langue maternelle française. Trois sujets étaient affectés de façon aléatoire à chacune des huit listes de matériel expérimental. Les protocoles individuels pris en compte lors de l'analyse des résultats ne comportaient pas plus de 6 erreurs de décision lexicale pour un total de 48 essais et pas plus de $35 \%$ de réponses incorrectes lors de l'épreuve de reconnaissance. Un sujet supplémentaire issu de la même population a participé à l'expérience afin de remplacer un sujet ayant systématiquement donné une réponse incorrecte dans l'une des conditions expérimentales.

\section{RÉSULTATS}

Les temps correspondant aux erreurs de décision lexicale étaient éliminés (4,5\% des essais expérimentaux), ainsi que les temps de décision supérieurs à la moyenne plus trois écarts types ou inférieurs à la moyenne moins trois écarts types $(2,3 \%$ des 
essais expérimentaux). Les temps moyens et les pourcentages d'erreurs de décision lexicale en fonction des conditions expérimentales sont présentés dans le tableau VII.

Tableat VII. - Temps de décision lexicale (en ms), écart type (entre parenthèses) et pourcentage d'erreurs en fonction de la fréquence relative, du mot amorce et du mot contexte. Expérience 3

Mean lexical decision latencies (in ms), standard deviations (in parentheses), and error percentage as a function of the relative frequency, the prime word and the context word. Experiment 3

\begin{tabular}{lcccc} 
& \multicolumn{3}{c}{ Fréquence relative de l'acception reliée au mot cible } \\
\cline { 2 - 5 } Mot contexte & $\begin{array}{c}\text { dominant } \\
\text { homographe }\end{array}$ & $\begin{array}{c}\text { mot amorce } \\
\text { non relié }\end{array}$ & $\begin{array}{c}\text { mot amorce } \\
\text { homographe }\end{array}$ & $\begin{array}{c}\text { mot amorce } \\
\text { non relié }\end{array}$ \\
\cline { 2 - 5 } relié & $567(108)$ & $600(101)$ & $580(96)$ & $603(105)$ \\
non relié & $6,9 \%$ & $4,2 \%$ & $2,8 \%$ & $5,6 \%$ \\
& $603(115)$ & $606(125)$ & $632(132)$ & $624(104)$ \\
\hline Effet du contexte & $4,2 \%$ & $1,4 \%$ & $6,9 \%$ & $4,2 \%$ \\
\hline
\end{tabular}

Deux analyses de variance ont été effectuées sur le temps moyen et le pourcentage d'erreurs de décision lexicale selon les plans : $S 24^{*} \mathrm{~F} 2{ }^{*} \mathrm{~A} 2{ }^{*} \mathrm{C} 2$ et $I 24<\mathrm{F} 2>{ }^{*} \mathrm{~A} 2{ }^{*} \mathrm{C} 2$, les lettres $S$, $I, F, A$ et $C$ renvoyant aux facteurs Sujets, Items cibles, Fréquence (dominant ou secondaire), Amorce (homographe ou non relié) et Contexte (relié ou non relié).

Les temps de décision sont plus courts lorsque le mot contexte est relié à la même acception que le mot cible que lorsqu'il est non relié $(F 1(1,23)=7,27, p<.05 ; F 2(1,46)=4,34$, $p<.05)$. Cet effet d'amorçage est plus important lorsque le mot amorce est l'homographe $(d=44 \mathrm{~ms})$ que lorsqu'il est non relié $(d=14 \mathrm{~ms})$. L'interaction entre les facteurs Contexte et Amorce n'atteint pas le seuil de signification dans les analyses par sujets et par items $(F 1(1,23)=1,91$, n.s. ; F2 $(1,46)=3,57$, n.s. $)$. Les interactions impliquant le facteur Fréquence ne sont pas significatives. Aucun effet principal et aucune interaction ne sont 
significatifs dans les analyses portant sur le pourcentage d'erreurs.

Des analyses portant sur les temps de décision lexicale ont été réalisées afin de tester directement l'effet du contexte relié à une acception dans chacune des conditions formées par le croisement des facteurs Fréquence et Amorce. L'effet du contexte correspond à la différence entre le temps de décision lexicale obtenu lorsque le mot contexte est relié à la même acception que le mot cible et le temps de décision obtenu lorsque le mot contexte est non relié. Lorsque le mot cible est relié à l'acception dominante, l'effet du contexte est significatif en présence de l'amorce ambiguë $(d=+35 \mathrm{~ms}, t 1(23)=2,84, p<.01$; $t 2(23)=2,17, p<.01)$, mais pas en présence du mot amorce non relié $(d=+7 \mathrm{~ms}, t 1<1 ; t 2<1)$. De même, lorsque le mot cible est relié à l'acception secondaire, un effet du contexte est constaté en présence de l'amorce ambiguë $(d=+52 \mathrm{~ms}$, $t 1(23)=2,45, p<.05, ; t 2(23)=1,72$, n.s. $)$, mais pas en présence du mot amorce non relié $(d=+21 \mathrm{~ms}, t 1(23)=1,09$, n.s. ; $t 2<1$ ).

En résumé, le contexte exerce un effet sur les temps de décision lexicale lorsque le mot amorce est l'item ambigu, mais pas lorsque le mot amorce est non relié. La présence de l'item ambigu est donc requise pour obtenir un effet du contexte. Ce résultat est compatible avec l'hypothèse selon laquelle les effets de contexte obtenus dans les expériences précédentes proviennent de l'effet du mot contexte sur l'accès à la signification de l'homographe, et non pas de la relation entretenue par les mots contextes et cibles reliés à la même acception. Par ailleurs, cette expérience a permis de retrouver l'effet de facilitation obtenu lorsque les mots contextes et cibles sont reliés à la même acception de l'homographe et constitue donc une réplique partielle des deux premières expériences.

\section{DISCUSSION GÉNÉRALE}

Dans les deux premières expériences que nous avons présentées, un couple de mots homographe-cible est précédé d'un mot contexte dont la nature est manipulée. L'évolution temporelle de l'effet exercé par ce contexte lexical est observée en faisant 
varier à la fois le temps de présentation des mots amorces et cibles et l'intervalle séparant la fin de la présentation du mot amorce du début de celle du mot cible. Le mot cible est relié à l'acception dominante de l'item ambigu dans la première expérience et à l'acception secondaire dans la seconde. Avec le SOA égal à $150 \mathrm{~ms}$, les temps de décision sont plus courts lorsque le contexte est relié à la même acception que le mot cible que lorsqu'il n'est pas relié, et ceci quelle que soit la fréquence relative de l'acception. La troisième expérience présentée a permis d'écarter la possibilité $d$ 'un effet direct du mot contexte sur le mot cible avec ce même SOA. L'effet du contexte lexical approprié persiste avec le SOA égal à $250 \mathrm{~ms}$, puis disparaît avec le soA égal à $400 \mathrm{~ms}$, illustrant ainsi la nature transitoire des phénomènes d'amorçage imputés aux relations entre les mots en l'absence d'un contexte phrastique sémantiquement et syntaxiquement cohérent (Lavigne-Tomps et Vitu, 1997). Ainsi, nous pouvons conclure qu'un contexte lexical formé d'un mot relié à une acception exerce un effet précoce sur l'accès à la signification des homographes. Cet effet du contexte ne remet pas en cause la conception modulariste. En effet, cette conception postule que la représentation intégrée d'un texte ne peut contraindre de façon précoce la résolution d'une ambiguïté lexicale, mais elle n'interdit pas des effets de contexte provoqués par des relations lexicales. En revanche, ces résultats ne sont pas conformes à l'hypothèse radicale d'un accès indépendant du contexte telle qu'elle a été formulée par Kintsch $(1988$, p. 171), pour qui, initialement, "seule importe la relation associative entre le mot amorce et le mot cible ». Il est à noter que cette hypothèse rejetant l'intervention précoce du contexte lexical sur l'accès à la signification a depuis été abandonnée (voir Kintsch, 1998).

Par ailleurs, aucun effet d'inhibition relative des temps de réponse n'est observé lorsque le mot contexte est relié à une acception différente de celle du mot cible, excepté lorsque le SOA est égal à $400 \mathrm{~ms}$ dans l'expérience 1 . Étant donné que cet effet d'inhibition n'est observé que relativement tardivement et n'est pas accompagné d'un effet $d u$ contexte lexical approprié, il a été interprété comme reflétant l'intervention de processus stratégiques intervenant après l'accès au lexique. Néanmoins, ces stratégies sont difficiles à préciser pour trois raisons. Premièrement, l'effet d'inhibition relative n'est pas observé avec les SOA 
les plus longs. Deuxièmement, il n'est jamais obtenu dans l'expérience impliquant le mot cible relié à l'acception secondaire de l'item ambigu, bien que les résultats concernant les pourcentages d'erreurs conduisent à une certaine prudence. Troisièmement, l'effet du contexte lexical approprié ne suit pas une évolution linéaire lorsque l'on considère les SOA les plus longs; lorsque le SOA est égal à $400 \mathrm{~ms}$, l'effet du contexte approprié n'est pas significatif. Cependant, il est à nouveau

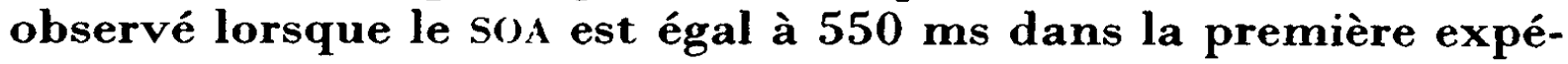
rience et lorsqu'il est égal à 450 et $700 \mathrm{~ms}$ dans la seconde expérience. L'ensemble de ces considérations nous conduisent à demeurer réservés quant aux interprétations des résultats obtenus avec un SOA supérieur à $250 \mathrm{~ms}$. Avec les SOA les plus courts, l'augmentation de l'activation d'une acception par le contexte ne s'accompagne pas de la diminution de l'activation de l'autre acception. Ce résultat est compatible avec l'hypothèse selon laquelle il n'existe pas de compétition entre les représentations relatives aux différentes acceptions d'un mot ambigu. Cette conclusion est à relativiser dans la mesure où elle repose sur un plan statistique sur une acceptation de l'hypothèse nulle. Cependant, il faut noter que les résultats des expériences 1 et 2 convergent vers cette conclusion et que celle-ci s'appuie sur d'autres résultats expérimentaux montrant un accès exhaustif aux différentes acceptions des homographes en l'absence de contexte relié à une acception.

Enfin, l'effet du contexte n'est pas modulé de façon précoce par la fréquence relative de l'acception à laquelle est relié le mot cible. Marquer et al. (1990) ont montré que les deux acceptions sont activées $120 \mathrm{~ms}$ après la présentation d'un item ambigu et que seule l'acception dominante demeure activée avec un SOA égal à $200 \mathrm{~ms}$. Ces résultats, obtenus en l'absence de contexte, ne sont pas contradictoires avec ceux obtenus dans les expériences 1 et 2 . Plus précisément, si l'effet du contexte sur l'accès aux acceptions dominante et secondaire est comparable pour les SOA de 150 et $250 \mathrm{~ms}$, cela ne préjuge pas d'un décours temporel différent pour l'activation de ces deux acceptions en l'absence de contexte.

Le principal objectif des expériences réalisées était de déterminer la façon dont sont représentés les mots homographes en mémoire. Les résultats obtenus sont compatibles avec l'hypothèse selon laquelle il n'existe pas de compétition entre les deux 
acceptions due aux caractéristiques structurales et fonctionnelles du lexique mental (Seidenberg et al., 1982; Twilley et Dixon 2000). L'acceptation de cette hypothèse n'est pas compatible avec celle des entrées lexicales multiples, dans la mesure où cette dernière suppose une compétition passive ou active entre les entrées. Cette dernière hypothèse semble couramment admise par les linguistes, pour qui deux homographes homophones sont deux mots différents qui partagent la même forme orthographique et phonologique, alors qu'un mot polysémique est un mot unique associé à plusieurs acceptions sémantiquement reliées. En faveur de l'hypothèse d'une entrée lexicale unique pour les homographes homophones, les résultats présentés remettent en cause la validité psychologique de la distinction opérée généralement en linguistique entre la polysémie et l'homonymie, du moins lorsque les différentes acceptions appartiennent à la même catégorie grammaticale. Cette distinction semble plutôt quantitative que qualitative, la différence résidant dans le degré de relation partagée par les différentes acceptions (voir Azuma et van Orden, 1997). 
ANNEXE. - MATÉRIEL EXPÉRIMENTAL UTILISÉ DANS LES 3 EXPÉRIENCES

\begin{tabular}{|c|c|c|c|c|c|c|}
\hline \multicolumn{3}{|c|}{ Mot contexte } & \multicolumn{2}{|c|}{ Mot amorce } & \multicolumn{2}{|c|}{ Mot Cible } \\
\hline dominant & secondaire & non relié & homographe & non relié & dominant & secondaire \\
\hline princesse & mâchoire & brin & palais & preuve & château & gô̂t \\
\hline souhait & photographie & apiculteur & objectif & province & but & lunette \\
\hline casino & aiguille & framboise & dé & ré & hasard & couture \\
\hline concours & achat & prisme & course & grappe & cheval & supermarché \\
\hline salon & toast & cylindre & canapé & psaume & fauteuil & apéritif \\
\hline école & adultère & cybernétique & maîtresse & poussière & institutrice & amant \\
\hline jeu & docteur & sabre & bridge & rideau & carte & dent \\
\hline conversation & nettoyage & chevreuil & entretien & vieillard & discours & ménage \\
\hline corps & combat & paquet & front & sœur & visage & guerre \\
\hline roman & diplôme & charcuterie & titre & honte & livre & récompense \\
\hline classement & meuble & ironie & dossier & ivresse & bibliothèque & chaise \\
\hline plante & vision & sénat & iris & gond & fleur & ail \\
\hline habit & trafic & viande & bretelle & escabeau & pantalon & autoroute \\
\hline herbivore & sandale & versement & mule & cube & âne & chausson \\
\hline chien & détective & segment & flair & limon & odeur & intuition \\
\hline ralcul & restaurant & fièvre & addition & occident & soustraction & note \\
\hline célébrité & port & fragmentation & vedette & uranium & star & bateau \\
\hline emprunt & extraction & sandalette & argent & groupe & banque & or \\
\hline sucre & terrasse & novice & café & рара & boisson & bar \\
\hline explication & ornement & axe & motif & jambe & raison & tableau \\
\hline sentiment & douleur & estafette & affection & président & amour & maladie \\
\hline blatte & tristesse & débat & cafard & citron & insecte & mélancolie \\
\hline cuivre & mal & moissonneuse & cor & suc & trompette & pied \\
\hline parent & piqûre & océan & cousin & empire & tante & moustique \\
\hline
\end{tabular}




\section{$R \hat{E} S U M E ́$}

Nous présentons trois expériences de décision lexicale réalisées afin d'étudier l'effet du contexte lexical sur l'accès à la signification des homographes homophones. Dans les deux premières expériences, chaque essai était constitué par la présentation d'un mot contexte, d'un item ambigu, et d'un mot cible. Les résultats ont mis en évidence un effet précoce du contexte sur l'accès à la signification. Alors que la présentation d'un mot contexte relié à la même acception de l'item ambigu que le mot cible entraînait une facilitation relative des temps de décision, un mot contexte relié à une acception différente ne provoquait pas d'inhibition relative du traitement du mot cible. Par ailleurs, cet effet du contexte se manifestait quelle que soit la fréquence relative de l'acception à laquelle était relié le mot cible. La troisième expérience nous a permis d'écarter la possibilité d'un effet direct du mot contexte sur le mot cible. La discussion de ces résultats porte sur la représentation des homographes en mémoire.

Mots-clés : représentation, ambigü̈té lexicale, homographes, contexte.

\section{BIBLIOGRAPHIE}

Azuma T., Van Orden (: - (1997) Why SAFE is better than FAsT' : The relatedness of a word's meanings affects lexical decision times, Journal of Memory and Language, 36, 484-504.

Balota D). A., Paul S. T. - (1996) Summation of activation : Evidence from multiple primes that converge and diverge within semantic memory, Journal of Experimental Psychology : Learning, Memory and Cognition, 22, $827-$ 84.5.

Borowsky R., Masson M. E. - (1996) Semantic ambiguity effects in word identification, Journal of Experimental Psychology : Learning, Memory, and Cognition, 22, 6.3-85.

Content A., Mousty P., Radeau M. - (1990) BRIIIfX : une base de données lexicales informat isée pour le français écrit et parlé, L'Année Psychologique, $90,531-566$.

Forster K. I. - (1976) Accessing the mental lexicon, in R. J. Wales et E. C. T. Walker (Edit.), New approaches to language merhanism, Amsterdam, North-Holland, 257-287.

Forster K. I. - (1979) Levels of processing and structure of the language processor, in W. E. Cooper et E. (. Walker (Edit.), Sentence processing : Psycholinguistic studies presented to Merrill Garrett, Cambridge, NIT Press, $27-$ 8.5.

Forser K. I. - (1981) Priming and the effects of sentence and lexical contexts on naming time: Evidence for autonomous lexical processing, Quarterly Journal of Fxperimental Psychology : Human Fxperimental Psychology, 3.3, $46.5-495$.

Forster K. I. - (1989) (On knowing how many entries, in I). S. (;orfein (Edit.), Resolving semantic ambiguit, New York, Springer-Verlag, 126-145.

Forster K. 1.. Bednall E. S. - (1976) Terminating and exhaustive search in lexiral aredess. Hemory and Cognition, f, 33-6].

françois. J. - (199.1) 1, a sémantique, in R. Ghiglione et J.-F. Richard (Edit.), Cours de Psvchologie, 3, Paris, Dunod. 5t-106. 
Gardye F. - (2000) Architecture structurale et fonctionnelle du lexique mental : les mots ambigus, thèse de doctorat de Sciences du langage, Université de Toulouse-Le Mirail.

Gernsbacher M. A. - (1984) Resolving 20 years of inconsistent interactions between lexical familiarity and orthography, concreteness, and polysemy, Journal of Experimental Psychology : General, I13, 256-281.

Gernsbacher M. A., Faust M. - (1991) The role of suppression in sentence comprehension, in G. B. Simpson (Edit.), Understanding word and sentence, Amsterdam, North-Holland, Elsevier Science Publishers, 217-240.

Glucksberg S., Kreuz R., Rho S. - (1986) Context can constrain lexical access : Implications for models of language comprehension, Journal of Experimental Psychology : Learning, Memory and Cognition, 12, 323-335.

Gottlob L. R., Goldinger S. D., Stone G. O., Van-Orden G. C. - (1999) Reading homographs : Orthographic, phonologic, and semantic dynamics, Journal of Experimental Psychology : Human Perception and Performance, 25, 561574.

Grainger J., Jacobs A. M. - (1996) Orthographic processing in visual word recognition : A multiple read-out model, Psychological Review, 10.3, 518-565.

Jastrzembski J. E. - (1981) Multiple meanings, number of related meanings, frequency of occurrence, and the lexicon, Cognitive Psychology, 1.3, 278-305.

Kellas G., Ferraro F. R., Simpson G. - (1988) Lexical ambiguity and the time course of attentionnal allocation in word recognition, Journal of Experimental Psychology : Human Perception and Performance, 14, 601-609.

Kintsch W. - (1988) The role of knowledge in discourse comprehension : A construction-integration model, Psychological Review, 95, 163-182.

Kintsch W. - (1998) Comprehension : A paradigm for cognition, Cambridge, Ciniversity Press.

Lavigne-Tomps F., Vitu F. - (1997) Time course of facilitatory and inhibitory semantic priming effects in visual word recognition, International Journal of Psycholinguistics, 13, 31 1-349.

Marquer P., Iebreton M., Léveillé M., Dioniso D. - (1990) À quel moment du traitement des homographes intervient la fréquence relative de leurs acceptions?, L'Année Psychologique, 90, 489-510.

Marquer P., Le Nestour J., Léveillé M., Welitz J. - (1982) Rôle de la fréquence relative des différentes acceptions d'homographes lors de l'accès au lexique, L'Année Psychologique, 82, 35.3-377.

Marslen-Wilson W. D., Tyler L. K. - (1987) Against modularity, in J. L. Garfield (Edit.), Modularity in knowledge representation and natural-language understanding, Cambridge, M I'T Press, 37-62.

Masson M. E. J. - (1995) A distributed memory model of semantic priming, Journal of Experimental Psychology : Learning, Memory and Cognition, 21, 3-23.

McClelland J. L., Rumelhart D. F. - (1981) An interactive activation model of context effects in letter perception : Part 1 . An account of basic findings, Psychological Review, 88, 375-407.

Mullet V. - (1994) Rôle de la polarité des homographes et de la nature du contexte dans l'accès au lexique, thèse de doctorat de psychologie, Université de Paris VIII.

Mullet V., Denhière G. - (1997) Accès au lexique et ambiguïtés lexicales nominales : effet de la polarité des homographes et de la nature du contexte, in J. François et G. Denhière (Edit.), Sémantique linguistique et psychologie cognitive. Aspects théoriques et expérimentaux, Grenoble, Presses Universitaires de Grenoble, 51-74. 
Neely J. H. - (1991) Semantic priming effects in visual word recognition, in i). Besner et (;. Humphreys (Edit.), Basic processes in reading : Visual word recognition, Hillsdale (..J), Erlbaum, 264-336.

Onifer W., Swinney I). - (1981) Accessing lexical ambiguities during sentence comprehension : Effects of frequency of meaning and contextual bias, Memory and Cognition, 9, 225-236.

Posner M. I., Snyder C. R. - (1975) Attention and cognitive control, in R. I. Solso (Edit.), Information processing and cognition, Hillsdale (N.J), Erlbaum, 5.5-85.

Rayner K., Pacht J. M., Duffy S. A. - (1994) Effects of prior encounter and global discourse bias in the processing of lexically ambiguous words : Evidence from eye fixations, Journal of Memory and Language, 3.3, 527-544.

Rubenstein H., I ewis S. S., Rubenstein M. A. - (1971) Homographic entries in the mental lexicon : Effects of systematicity and relative frequency of meanings, Journal of Verbal Learning and Verbal Behavior, 10, 57-62.

Schvaneveldt R. W., Meyer D. E., Becker C. A. - (1976) Lexical ambiguity, semantic context and visual word recognition, Journal of Experimental Psychology : Human Perception and Performance, 2, 24.3-256.

Segui J. - (1992) Perception du langage et modularité, in D. Andler (Edit.), Introduction aux sciences cognitives, Paris, Gallimard, «Folio Essais 》, 131 152.

Seidenberg M., Tanenhaus M., Leiman J., Bienkowski M. - (1982) Automatic access of the meanings of ambiguous words in context : Some limitations of knowledge-based processing, Cognitive Psychology, 14, 489-537.

Sharkey N. E. - (1990) A connectionnist model of text comprchension, in D. Balota, G. B. Flores d'Arcais et K. Rayner (Edit.), Comprehension processes in reading, Hillsdale (N.J), Erlbaum, 487-511.

Simpson G. B. - (1981) Meaning dominance and semantic context in the processing of lexical ambiguity, Journal of Verbal Learning and Verbal Behavior, $20,28-39$.

Simpson G. B., Burgess C. - (1985) Activation and selection process in the recognition of ambiguous words, Journal of Experimental Psychology: Human Perception and Performance, 11, 28-39.

Tabossi P. - (1988) Accessing lexical ambiguity in different types of sentential context, Journal of Memory and Language, 27, 324-340.

Tabossi P., Colombo L., Job R. - (1987) Accessing lexical ambiguity : Effects of context and dominance, Psychological Research, 49, 161-167.

Tabossi P., Zardon F. - (1993) Processing ambiguous words in context, Journal of Memory and Language, 32, 359-372.

Thérouanne P. - (2000) Effet du contexte lexical sur l'accès à la signification des homographes polarisés, thèse de doctorat de Psychologie, Lniversité d'AixMarseille I.

Till E., Mross F., Kintsch W. - (1988) Time course of priming for associate and inference words in a discourse context, Memory and Cognition, 16 (4), 283298.

Twilley I.. C., Dixon P. - (2000) Meaning resolution processes for words : A parallel independent model, Psychonomic Bulletin and Review, 7, 49-82.

Vu II., Kellas G., Paul S. T. - (1998) Sources of sentence constraint on lexical ambiguity resolution, Memory and Cognition, 26, 979-1001.

(Accepté le 11 mai 2001.) 